\title{
In-Vitro Evaluation of Amlodipine Tablets Available in Nepalese Market
}

\author{
Pabitra Thapa, Nayan Manandhar and Shiva Pandeya* \\ Institute of Medicine, Tribhuvan University, Maharajgunj Medical Campus, Nepal \\ Email: shivapandeya@iom.edu.np
}

\begin{abstract}
Physicochemical properties of 11 brands of the amlodipine besylate $5 \mathrm{mg}$ tablets were assessed according to official and unofficial standards. Basic quality control parameters evaluated include uniformity of weight, uniformity of content of active ingredients, hardness test, and disintegration and dissolution tests as per pharmacopoeial requirement of Indian Pharmacopoeia (IP) 2007. The results show that all the tablets passed the weight variation test, friability ascertain the presence and compendia quality of amlodipine besylate in all these selected sample. The invitro evaluation parameters profiles were found to be varying for each brand, but within the standard limit. The disintegration time of the samples varied from 01:30 to 03:50 minutes. The content of Amlodipine was observed within $97.8933+1.16367 \%$ to $103.0+2.0 \%$ of claimed amount of $5 \mathrm{mg}$ per tablet. The average dissolution \% of Amlodipine tablets $(n=11)$ was observed from $77.2 \%$ to $96.85 \%$. The average dissolution percent of all the sample tablets were observed within the standard requirement of not less than $70 \%$.
\end{abstract}

Keywords: Physicochemical properties, Amlodipine Tablets, World Health Organization

\section{Introduction}

The quality of medicine concerns both health officials \& general public; frequently questions are raised regarding the quality of pharmaceutical products in the market. Pharmaceutical products play an important role in improving the health and promoting the well- being of every individual ${ }^{1}$. These medicines aid in the prevention and treatment of diseases, disorders, or conditions. These agents can provide relief of symptoms and favorably modify the course of diseases. The three criteria considered the cornerstone of these products- quality, effectiveness and safety, should be demonstrated and verified prior to their rational $u^{2} \mathrm{e}^{2}$. Same medicines of different manufacturers may have variable responses due to formulation ingredients employed, methods of handling, packaging, and storage practices. Thus there is need to determine the pharmaceutical and therapeutic equivalence in order to ensure interchangeability ${ }^{3}$. However, developing countries like ours do not have an effective means of monitoring the quality of products in the market, these results in wide spread distribution of substandard and/or counterfeit drug product. It was in a view of this fact that the World Health Organization (WHO) issued guidelines for global standard and requirements for the registration, assessment, marketing authorization and quality control of pharmaceutical products ${ }^{3,4}$.

${ }^{*}$ Corresponding author 
Counterfeit drugs are those which are deliberately or intentionally \& fraudulently mislabeled with respect to identity and/or source. Substandard drug on the other hand are those produced by legitimate manufacturers but don't meet pharmacopoeial standard ${ }^{5}$. It is estimated that up to $25 \%$ of the medicine marketed in the developing countries are counterfeit or substandard. This counterfeit $\&$ substandard drug is becoming a great threat in public health \& quality of health care system. ${ }^{6}$ High blood pressure is estimated to cause 7.1 million deaths, about 13 percent of the global fatality total. It is believed this number will grow to approximately 11 million by the year 2020. Hypertension is the leading risk factor for cardiovascular and renal disease, increasing the risk of myocardial infarction, stroke, congestive heart failure, ruptured aortic aneurysm, and renal disease ${ }^{7}$. Amlodipine is a calcium channel blocker, which in addition to its anti-angina and anti-arrhythmic effects also dilates peripheral arterioles and reduces blood pressure. This multifaceted function in mediating cardiac activity has led to its popularity in terms of its wide acceptance and use in hypertension management ${ }^{8}$. The detail information on the drug studied in this work is provided below.

Name:

Chemical name:

Molecular weight:

Empirical formula:
Amlodipine Besylate

(RS)-3-ethyl 5-methyl 2-[(2-aminoethoxy) methyl]-4-(2chlorophenyl)-6-methyl-1, 4-dihydropyridine-3, 5-dicarboxylate

567.1

$\mathrm{C}_{20} \mathrm{H}_{25} \mathrm{CIN}_{2} \mathrm{O}_{5}$

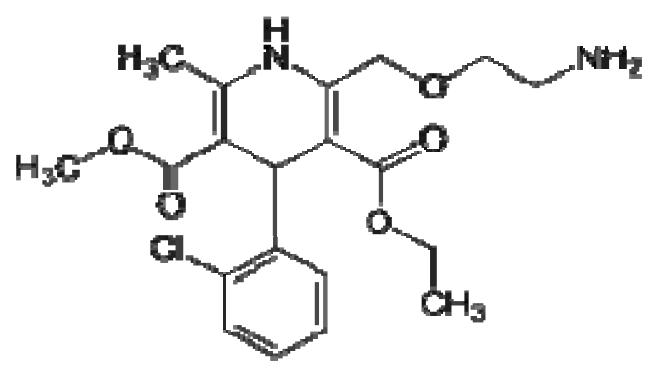

Figure 1: Chemical structure of Amlodipine

\section{Experimental Methods}

The samples (brands) were purchased from the market and coded as A-01 to A-11. The samples were then analyzed according to IP and the results obtained were analyzed with application of statistical methods.

\section{Weight variation test}

The percentage of weight variation was determined as the upper and lower deviation by using the following relation ${ }^{9}$

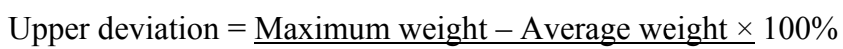

Average weight 


\section{J. Nepal Chem. Soc., Vol. 39, 2018}

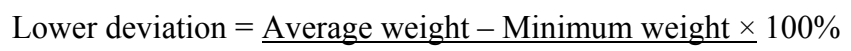

\section{Average weight}

According to pharmacopoeia, following limits are given for the weight variation of tablets.

Table 1: Pharmacopoeial limit for weight variation of tablets

\begin{tabular}{|l|l|}
\hline Average weight & \% Deviation \\
\hline $80 \mathrm{mg}$ or less & $\pm 10 \%$ \\
\hline More than $80 \mathrm{mg}$ but less than 250mg & $\pm 7.5 \%$ \\
\hline $250 \mathrm{mg}$ or more & $\pm 5 \%$ \\
\hline
\end{tabular}

Note: Since the sample to be studied was $5 \mathrm{mg}$, the deviation limit should be within $10 \%$ range .

\section{Hardness Test}

Hardness of eleven tablets of each brand was tested with the use of hardness tester. Hardness is measured in kilogram per $\mathrm{cm}^{2}$. Minimum hardness of $4 \mathrm{~kg} / \mathrm{cm}^{2}$ is required for the tablet to be satisfactory $^{15}$.

\section{Disintegration Time}

Disintegration time of the tablets was determined by the use of tablet disintegration test apparatus (Thermonik - Campbell Electronics, India).One tablet was introduced into each tube and the disc was placed into each tube. The whole assembly was suspended in the beaker containing distilled water at $37 \pm 2^{\circ} \mathrm{C}$. The apparatus was operated until no residue remained on the screen or adhered to the inner surface of the disc and the disintegration time was noted.

\section{Table 2: Pharmacopoeial limit for disintegration time of tablets}

\begin{tabular}{|l|l|}
\hline Tablet type & Disintegration time \\
\hline Uncoated tablet & Not more than $15 \mathrm{mins}$ \\
\hline Film coated tablet & Not more than $30 \mathrm{mins}$ \\
\hline Enteric coated tablet & Not more than $45 \mathrm{mins}$ \\
\hline
\end{tabular}

Note: Since the sample to be studied was film coated, the disintegration time should not be more than 30 minutes 9 .

Assay

The International Pharmacopoeia suggests an HPLC method for the analysis of Amlodipine in tablet formations; however, the use of a spectrophotometric method has also been reported. Thus, the spectrophotometric method was used for the assay determination of Amlodipine tablets. The pharmacopoeial limit for the content of Amlodipine is (90-100) \%.

\section{Dissolution Test}

The dissolution test was carried out using the Dissolution Rate Test Apparatus (Thermionic Campbell Electronics, India). The dissolution parameters were: Medium, 0.01M hydrochloric acid $(900 \mathrm{ml})$ at $37 \pm 0.5^{\circ} \mathrm{C}$; Apparatus, paddle type; Rotational speed, $75 \mathrm{rpm}$; Time, $45 \mathrm{mins}$. The suitable 
volume of solution was withdrawn at specified time and filtered through filter paper. The absorbance of the resulting solution was observed at $\lambda_{\max } 239 \mathrm{~nm}$ taking ethanol as a blank. The pharmacopoeial limit for the dissolution of amlodipine tablets is not less than $70 \%$ of the stated amount of $\mathrm{C}_{20} \mathrm{H}_{25} \mathrm{CIN}_{2} \mathrm{O}_{5}{ }^{9}$.

\section{Results and Discussion}

The hardness of the samples varied from maximum of $9.0 \mathrm{~kg} / \mathrm{cm}^{2}$ to minimum of $3.0 \mathrm{~kg} / \mathrm{cm}^{2}$. Sample A-03 has maximum hardness of $9.0 \mathrm{~kg} / \mathrm{cm}^{2}$ while sample A-04, A-06, A-09 and A-11 had a minimum hardness of $3.0 \mathrm{~kg} / \mathrm{cm}^{2}$.

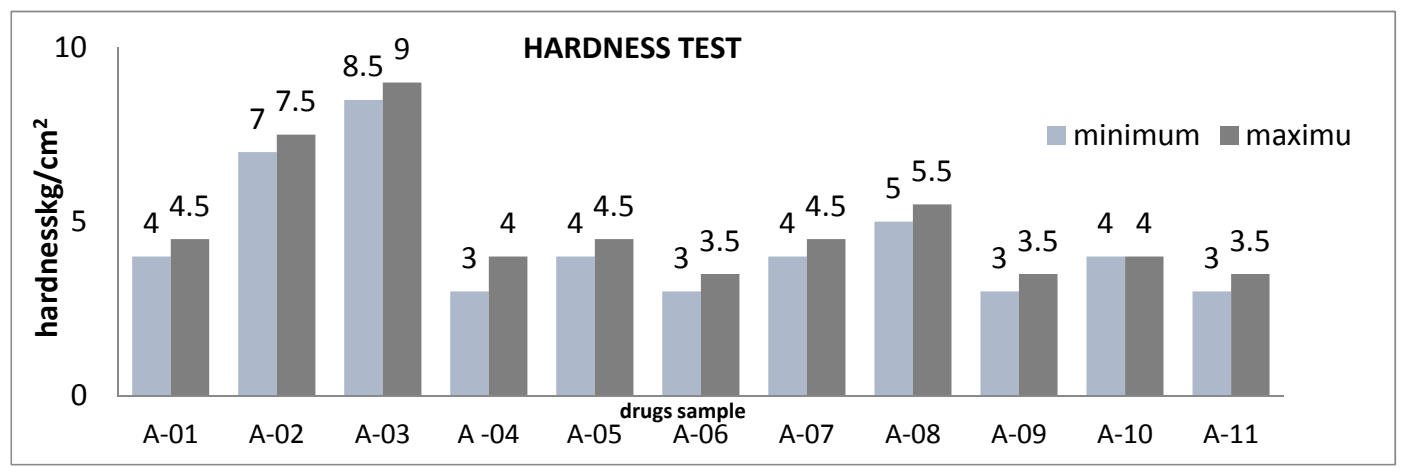

Figure 2: Hardness test of the samples.

The disintegration time of the samples varied from 01:30 to 03:50 minutes. Samples A-03 showed minimum disintegration time of 01:30 minutes and the sample A-05 showed maximum disintegration time of 03:50 minutes.

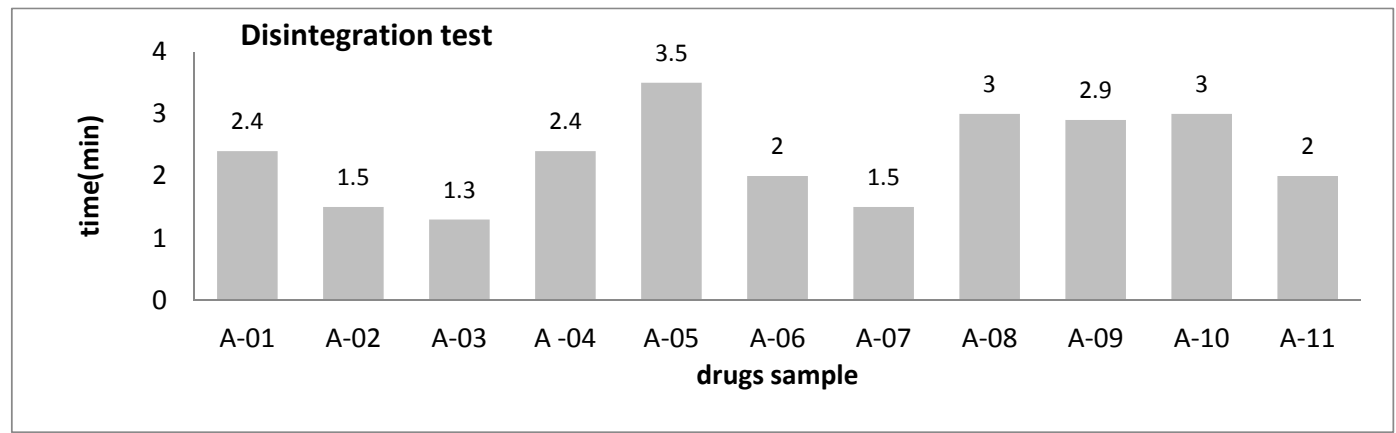

Figure 3: Disintegration test of the samples.

The weight of the tablets varied from 0.0583-0.2019 $\mathrm{g} /$ tablet. Among the samples studied all the samples passed the weight variation test according to the pharmacopoeial requirement. 


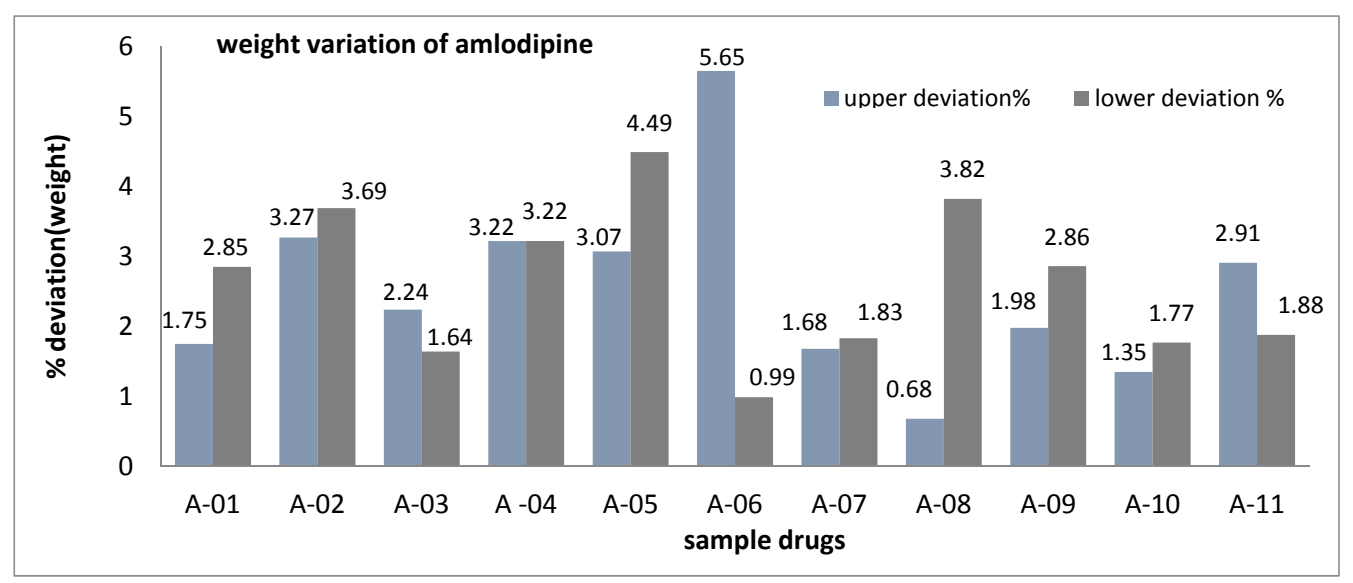

Figure 4: Weight variation test of the sample

The content of Amlodipine was observed within $97.8933 \pm 1.16367 \%$ to $103.0 \pm 2.0 \%$ of claimed amount of $5 \mathrm{mg}$ per tablet.

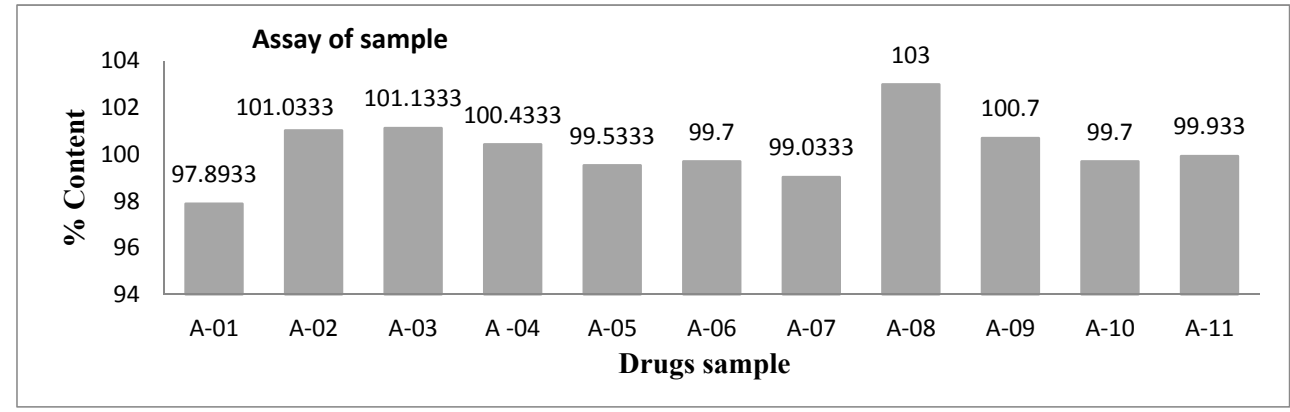

Figure 5: Assay for the content of active ingredient of the samples

The average dissolution \% of Amlodipine tablets ( $\mathrm{n}=11$ ) was observed from $77.2 \%$ to $96.85 \%$. The average dissolution percent of all the sample tablets were observed within the standard requirement of not less than $70 \%$.

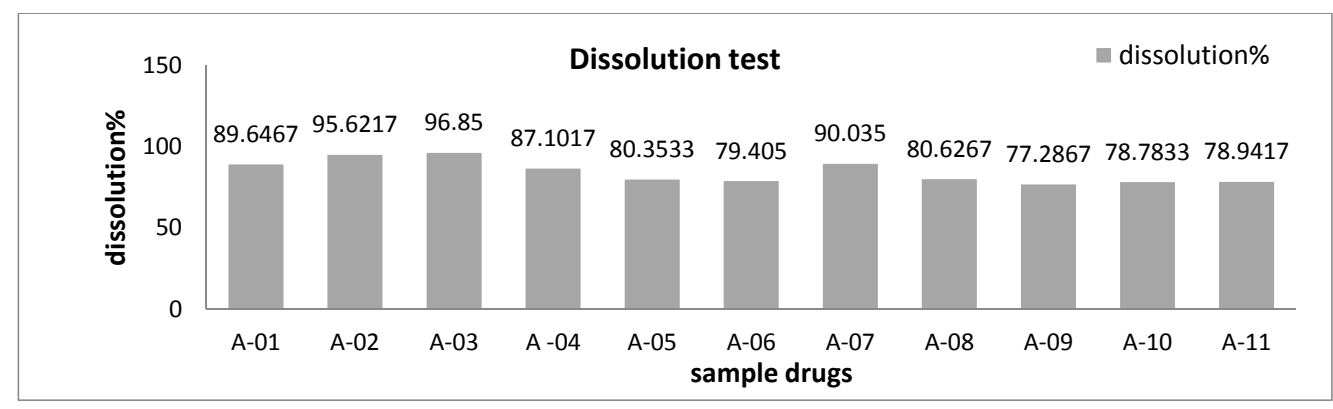

Figure 6: Dissolution test for the sample 
The result of content uniformity test of the sample showed that all the samples had content of amlodipine within the present pharmacopoeial limit.

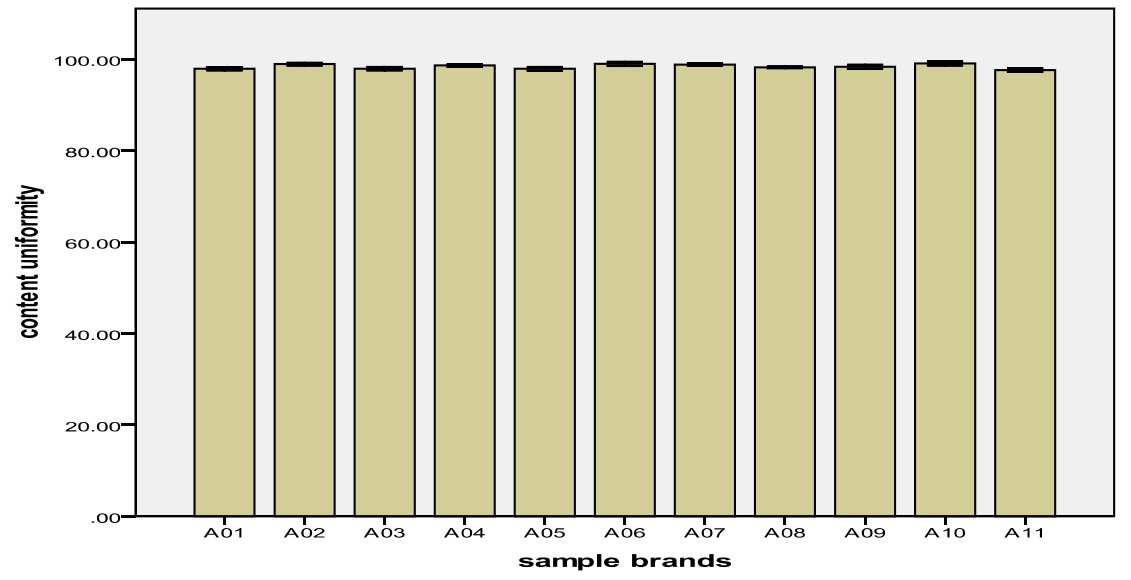

There were some variations between tablets with regards to hardness. Hardness of a tablet is a function of the die fill and the compression force. The hardness of tablets is an essential criterion in the determination of the ability of the tablets to resist chipping, abrasion or breakage under conditions of storage, transportation, and handling before use, and it is also associated with other tablet properties such as density and porosity. All samples passed the minimum strength that the tablets should possess to resist abrasion, chipping or breakage under conditions of storage, transportation or handling. ${ }^{10}$ The disintegration time of all brand were within the standard limit for film coated tablets. Disintegration is the first step of drug release process where tablets disintegrates into smaller fragments and becomes available for dissolution in the medium. All the brands pass the weight variation test according to pharmacopoeial requirements. Weight variation test is required to ensure that the drug content in each unit dose is distributed in a narrow range around the label claim. Weight of the tablets may be varied due to problems in granulation and compression during the manufacturing process. All the samples had the content of amlodipine within the standard limit hence all the sample passed the content uniformity test. The average dissolution percentage of all the samples of amlodipne tablets were observed within the standard requirement of not less than $70 \%$.

\section{Conclusions}

The research work find out that all the selected brands confirmed the pharmacopoeial specifications. Thus the study concludes that the selected brands of amlodipine Besylate tablets available in Nepalese market are of required quality in accordance with the pharmacopoeial standard. Numbers of brands of amlodipine are available in Nepalese market with variable cost price. Since the study has shown standard of all tested sample as per requirements and specification, prescribers can choose the brand on benefit of patient as per cost and availability. This study has also confirmed the standard of domestic drugs along with multinational company as all the sample from the domestic company has met the pharmacopoeial and non-pharmacopoeial requirements. Thus from this research it could be concluded that there is no 


\section{J. Nepal Chem. Soc., Vol. 39, 2018}

significant difference between various brand of amlodipine besylate tablets (domestic as well as multinational) in terms of quality assurance.

\section{References}

1. United States pharmacopoeia (USP). A review of drug quality in Asia with focus on antiinfective. United States: Inc. Rockville, MD, 2004.

2. Quality assurance Available from: http:/www.who.int/medicines/areas/quality-safety/qualityassurance/en/index.html (Oct. 2011).

3. World Health Organization. Multisource products: Guidelines on registration requirement to establish inter changeability. Geneva: WHO headquarter; 2004: p. 1-5.

4. Counterfeit medicines. Available from: http://www.ffd.gov/drugs/resourse for you/cnsumers/ buying using medicine safely/counterfeit medicine/default.html

5. World Health Organization. WHO policy perspectives on medicines- effective medicines regulation: ensuring safety, efficacy and quality. Geneva: WHO headquarter, 2003.

6. World Health Organization, Substandard and counterfeit medicines. Geneva: WHO headquarter fact sheet no. 275 .

7. P. K. Whelton, J. He J, J. Appel, Primary prevention of hypertension: Clinical and public health advisory from the national high blood pressure education programme. JAMA 2002; pp. 288.

8. B. G. Katzung, K. Chatterjee K. Basic and Clinical pharmacology. $9^{\text {th }}$ edition. New York: Mcraw Hill Companies; 2004: pp. 184-199.

9. Indian Pharmacopoeia (IP). Volume- I. Govt. of India, Ministry of Health and Family Welfare. Delhi: Controller of Publications; 1996

10. B M. Mittal, A textbook of pharmaceutical formulation. $6^{\text {th }}$ edition. Delhi: Vallabh prakashan; 1997. 